\title{
Service Broker-Based Architecture Using Multi-Criteria Decision Making for Service Level Agreement
}

\author{
Imane Haddar ${ }^{1}$, Brahim Raouyane ${ }^{2} \&$ Mostafa Bellafkih $^{1}$ \\ ${ }^{1}$ Institut National des Postes et Télécommunications, Rabat, Morocc \\ ${ }^{2}$ Faculty of Sciences Ain Chock, Casablanca, Morocco \\ Correspondence: Imane Haddar, Institut National des Postes et Télécommunications, STRS Lab, Rabat, \\ Morocco.
}

Received: November 4, 2019

Accepted: December 19, 2019

Online Published: December 24, 2019

doi: $10.5539 /$ cis.v $13 \mathrm{n} 1 \mathrm{p} 20$

URL: https://doi.org/10.5539/cis.v13n1p20

\begin{abstract}
With the on-going trends of the telecom services, the number of service providers with similar functionalities is undergoing a rapid growth. The customers face the difficulty to decide which service provider can satisfy their needs and full their requirements. Negotiating contracts between involved parts, and hiding heterogeneity in the distributed network environment has been challenging for telecom operators and service providers. Different languages exist to describe the Service Level Agreement (SLA), which is a contract between a service provider and a customer. However, since each service provider expresses his SLA in his own way, it disrupts the customer's choice of the best service provider, and leads to a bad contract management. In this respect, we propose a novel architecture for service selection, and SLA management between different stakeholders in our network architecture. The idea is to set up a smart broker where we implemented a Multi-Criteria Decision Making (MCDM) method to maximize utility function so that the customer can choose services with required QoS performances. We also came up with the idea of settling a negotiation model for the SLA, and a context based SLA contract ontology in IP Multimedia Subsystem (IMS) network is also proposed to provide users with a clear model to express their requirements and preferences. Moreover, we used the New Generation Operations Systems and Software (NGOSS) Framework to model and analyze networks and services actions. To better understand the relationship and the projection of NGOSS Framework and IMS platform, we introduce an SLA management and monitoring architecture in IMS network.
\end{abstract}

Keywords: service level agreement, service broker, multi criteria decision making, enhanced telecom operations map

\section{Introduction}

With the existence of Telecom operators; and third-party telecom services, millions of applications/services are becoming tens of millions. Consumers are always searching for services that respond to their functional and non-functional properties to maximize their utilities. Most of the time, consumers faced the inevitability of selecting the right service that satisfies their needs. The utility of using a specified service obviously depends on the QoS offered by the service provider. Several researchers proposed approaches as a remedy for the service selection problem. However, they did not take into consideration the relationship between consumers' expectations and the utility a consumer derives from a service. A lot of challenges exist in service selection, starting with how a consumer defines his preferences when it comes to QoS parameters, because each consumer has his own requirements, another issue is related to the consumer objectives, for instance, response-time of a number of seconds can be acceptable in a context, and not acceptable in another. Due to the changes that a service can undergo, and because of restricted knowledge of consumers about services, the selection of the right service is clearly not an easy task. The success of any service provider depends especially on the consumer satisfaction which is related to the service performance and the QoS. Service providers and consumers agreed on different issues associated to QoS in the SLA contract. The SLA contract contains all the agreements about the type the service to provide, the service cost, the level of the QoS to deliver, and the penalties to impose on the service provider in case on violation of the agreement. Different approaches of service selection show the path for consumers to select the right service without take into consideration consumers' requirements. However, the best approach should guide consumers to the best service based on their preferences and old SLAs proposed by 
service providers. For this purpose, our approach addresses all the issues cited above. It incites to collect all the information about the service, it also urged consumers to indicate their preferences, and eventually, our approach will maximize the utility of each QoS attribute requiring maximization (e.g. availability), and minimize the utility of other QoS attributes that require minimization (e.g. the cost). Given also the wide variety of services offered by different Telecom service providers, consumers are facing other challenges because of the heterogeneity of different offers, and how to formulate a coherent service request. For this reason, our approach consists on checking the consistency of each consumer request.

Also, knowing the rapid evolution of the telecommunication industry and for telecom service providers to be distinguished, improved management architecture should be offered to ensure consumer loyalty. Evenly providers give more attention to their reputation so the management task is an important point for them to maintain a good impression and keep a proper image. For this purpose, we propose management architecture to monitor the network, and our proposition aims at offering an efficient dynamic service discovery and selection solution. Having different units when using different criteria motivate the use of multicriteria decision aid methods to solve the kind of issues mentioned above. Furthermore, the syntactic and se- mantic heterogeneity between LTE service providers make this choice increasingly difficult. This heterogeneity is corroborated by expressing the same notion in different ways using different terminologies, also, formulating the consumer request by various languages, and expressing the quality of service in different ways, for instance, two different metrics can have the same while expressing the same concept such as the word delay can refer to the latency of a system as it can express the percentage of packets lost during the transmission of a service mentioned by Haddar et al., (2017).

In this paper, we describe our architecture for telecom service selection. The principal component is the Service Broker which takes in charge the selection of the required service by the consumer, checks the coherence of the request, and manages the network mechanism.

The rest of this paper is organized as follows. The next section gives an overview about the selection concept and presents a review of related works. In section 3, our proposed approach for service selection, negotiation and SLA ontology is discussed in detail, including the architectural component and interfaces used in our architecture. Section 4 describes the simulation result of our testbed model. Managing the network is well described in section 5. Section 6 sums up the article with a few concluding remarks.

\section{Background and Related World}

\subsection{Service Selection Methods}

After finding a service, the selection stage comes just after. A lot of researchers proposed different techniques to help consumers or requesters in general to choose and select the service that most meet the consumer requirements. Service selection is considered as an important step in telecom service lifecycle and has been a major work of computing and telecom researchers. Some researchers rely on the size of the problem to solve the issue, for instance Parra-Hernandez and Dimopoulos (2005) precise that they have to use heuristic approaches to solve their problem given that they face an NP-hard problem. Numerous heuristic algorithms for service selection exist, for example, those explained by Berbner et al., (2007) and Menascé et al., (2010). Garg et al., (2011) proposed a framework, comparing and ranking services based on a set of defined attributes. Wang et al. (2011) proposed a model that computes QoS and find the suitable service using mixed integer programming. Other researches done by Ur Rehman et al., (2012) use MCDM to select a cloud service; they proposed an approach of a system that use old information to select the best offer. In our work, we focused on MCDM method due to its advantages. It is used when it comes to solve problems with multiple objectives. Different MCDM methods exist; the most applied techniques are: 1) the outranking approaches like ELECTRE (Bouyssou, 2009; Roy, 1991), also PROMETHEE detailed by Brans and Mareschal (1990). 2) Compensatory Method, for instance Analytical Hierarchy Process (AHP) detailed by Brans and Mareschal (1990), it is the most recognized example of Compensatory MCDM, this method de-composes a decision problem into a set of graduated levels, and help decision makers to select the solution that best suits the request. Finally, 3) Multi-criteria value functions or MAUT (Multi Attribute Utility Theory) detailed by Keeney and Raiffa (1993) is an approach used for choosing the most wanted alternative among numerous alternatives, it has been involved in different fields, it is defined by Dyer (2005), and Ishizaka and Nemery (2013) as a utility theory solving complicated problems with various objectives requiring making decision. Many researchers used the MAUT approach to validate their theory as reported by Fishburn (1967), the form of the utility function can be in an additive or a multiplicative form. Telecom services of the same type may provide the same functionality, but these features may vary depending on the values of the quality of service attributes that are generally defined by the service functionality 
(O'sullivan et al., 2002), and are classified as follows: Technical attributes, like response-time, reliability, and availability. Regarding the availability attribute as we defined in our previous work, it represents the rates of how to access to a service any time. However, reliability represents the degree of maintaining the service and the ability of a service to achieve the different requested tasks and functions. Response-time, which quantify how long the user must wait for a response to a query, and also the charges and the price of a requested service called a cost (Haddar et al., 2017).

Non-technical attributes, such as: penalties, image and reputation of a service provider. As mentioned by Medjahed and Atif (2007), service providers offer the same type of service but with different level of quality of service depending on the consumers' requests which differ from one another because of the preferences and the constraints of each consumer.

\subsection{Negotiation}

In different field and in any normal process, the negotiation phase is considered as a very important step between the consumer and the service provider to settle an agreement and resolve conflicts. Lot e orts have been made to solve the SLA negotiation issues. The standard form of a negotiation is about one problem like mentioned by Lee and Ferguson (2010), however, in real life and especially in telecom domain, the stakeholders have to negotiate different attributes to reach mutually an interesting outcome, surely, this type of negotiation is time consuming because of the different consumer preferences that a service provide has to respect as stated by Lai et al., (2006). With the tremendous growth of the use of telecom services, generated negotiation systems are increasingly used to negotiate on behalf of the consumer and take the best deal of SLA with the service provider (Chen and Huang 2009; Chevaleyre et al., 2006). For example, authors in Di Modica et al., (2009) show that the use of WS-Agreement to specify the SLA contract, and they proposed new functionalities to improve their framework. Silaghi et al., (2012) had a different vision; they proposed a new system for implementing an automatic negotiation framework. Their framework is based on old information from negotiation relies on learning opponent strategies that at the end lead to consumer and service provider satisfaction. In another work, Wu et al., (2013) proposed a framework for automated contracts with several service providers. The negotiation system is based on heuristics methods to generate service providers' propositions. This method relies on the trade-o between QoS parameters and the different constraint in the world of telecom service. However, Dan et al., (2004) proposed a frame- work based on service oriented architecture with SLA and automated management. Their system allocates resources dynamically.

\subsection{Ontology}

Describe the procedures for selecting participants, including (a) the sampling method, if a systematic sampling plan was used; (b) the percentage of the sample approached that participated; and (c) the number of participants who selected themselves into the sample. Describe the settings and locations in which the data were collected as well as any agreements and payments made to participants, agreements with the institutional review board, ethical standards met, and safety monitoring procedures.

The problem that faces consumers is related to the heterogeneity of the offers. Different efforts have been made to enhance the selection of requested services using the concept of ontology. Ontology as defined by Bermejo (2007) is used in different fields due to its benefits. It contributes essentially to identify the contract between the involved parties, in our case, between service provider and consumer, this contract is called SLA, it is a legal document that contains different information about the service, like performance, billing, activation-time, service-level objectives (SLOs), and penalties in case of any violation of the service like explained by Baset (2012), and Dan et al., (2004).

Actually, each service provider describes the contract in his own way, using not necessarily the same language. Some efforts to standardize the use of SLAs have been developed by Web Service Level Agreement (WSLA) by IBM Keller and Ludwig (2003).

WS-Agreement specification Andrieux et al., (2007), as well Lamanna et al., (2003) and Lai et al., (2006) stated the SLAng Specification Language in their works. That is why the consumer gets confused about the choice of the right service provider. Another issue about the current structure of the proposed ontology arises because of the non-conformity of any standardization efforts. Different propositions have been settled to make the selection of the service an easy task with the use of ontology that helps to upgrade the service selection for the consumer request Fakhfakh (2015).

Different researchers concentrated on ontology to allow functionalities such as service description, service discovery, and so on. They specified that ontologies are made to find services in different domains, such as cloud 
domain and web service domain. Authors in Ma et al., (2011) proposed ontology based on resources information and agreed SLAs. They have proved that ontology improves the ability and the capability of the resource management structure. Other authors proposed a management method for service composition in mobile internet network. To describe their scenario and the component, they used Ontology Web language for services (OWL-S, which is essentially composed of service prole where the service is described in details, the process model which contains the interactions with the service, and last, the details about the interactions with the service.

Tarkoma et al., (2007) used some technologies with ontology to manage the integrated requirements in mobile service platforms; they invoked the dynamic adaptability to user demands and conditions. Sulaiman et al., (2017) proposed to use ontology to minimize the semantic gap by expending multi-modality ontology image retrieval with the reinforcement of recuperation action using the object properties filter.

From all these and other researches, the models proposed and executed by ontology system do not meet services behaviours and their definition. The service structure must be updated and adapted in a natural way to ensure the interoperability of LTE $/ 5 \mathrm{G}$ services with the network platform. Up to the writing of this article there are no contributions research based on ontology for LTE/5G architecture. But it should be mentioned that in the 5G, a new QoS framework with flows is introduced rather than end-to-end bearers, and new approach to $5 \mathrm{G}$ core network using service based architecture concept.

Despite all researches that propose methods for service selection and negotiation, few of them proposed approaches that rely on brokers, such as Hasselmeyer et al., (2006) who proposed a broker based framework for a contract negotiation, the broker negotiate on consumers' behalf. However, Wu et al., (2013) and as mentioned before, proposed a broker architecture for contract negotiation and their method is based on finding every consumer needs to respond to his request using heuristics methods.

Concerning our work, we aim to provide a dynamic Service Broker for service selection, SLA negotiation, and architecture management. Our Service Broker helps first all consumers to formulate their requests in a consistent way using ontology model, it hides heterogeneity in a network environment, and it also select the right service provider with whom negotiate the SLA contract and manage the network.

\section{Proposed Architecture}

Our proposed architecture is a dynamic one where we can find combined contents with the ontological model. It incorporates semantic knowledge along with different service criteria. Our test bed relies different component among then IMS (IP Multimedia Architecture) entities on which we did our test bed. Figure 1 illustrated by Mendiratta and Pant (2007) shows the IMS Architecture.

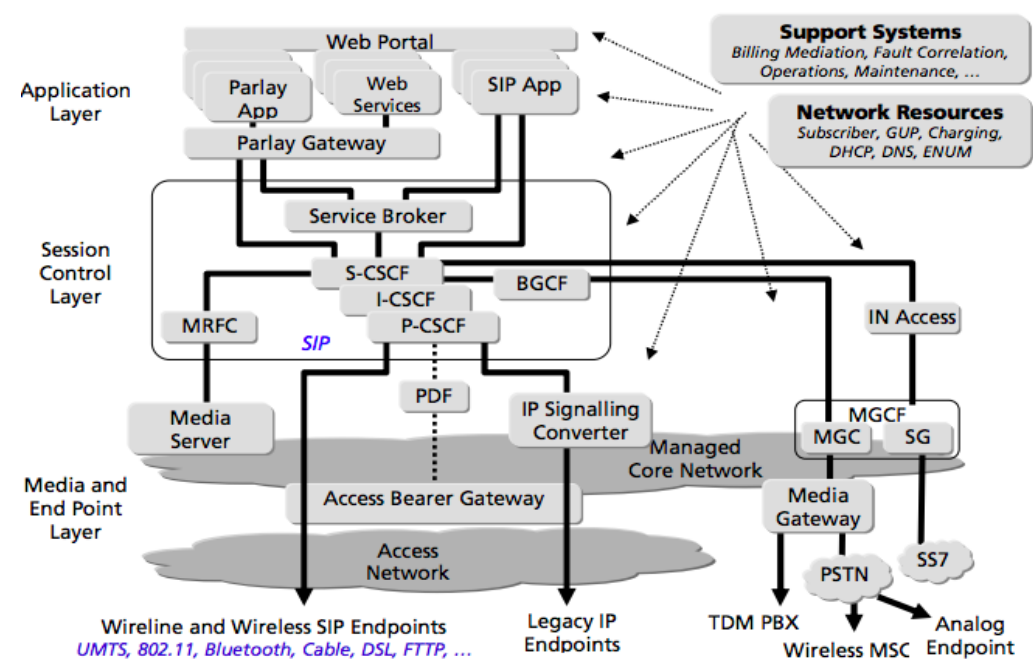

Figure 1. IMS network architecture

The IMS is an architecture framework for delivering IP multimedia services. The IMS standard is proposed by 3GPP Navarro et al., (2010). The IMS architecture is a layer network ranging from access layer to service layer passing through the transport/control layer which is the heart of the architecture, it contains the Call Session Control Function (CSCF), which allows the endpoints for the routing and the registration for the Session Initial 
Protocol signaling messages, enabling them to be delivered with the correct application services.

The architecture includes also the database that contains different information about subscribers, this database is called Home Subscriber Server (HSS) (Navarro et al., 2010), and it includes the user preferences as well as their registration details.

The focus of our work goes to the service selection, and like we mentioned before, our selection approach is done based on MCDM methods, where we use Visual PROMETHEE software. In any situation, decision-makers or stakeholders have several objectives, so they have to compare different existing solutions called actions. For instance, for Key Performance Indicators, costs should be minimized; profit and efficiency should be maximized, etc. Defining appropriate quantitative or qualitative evaluation criteria can help to measure the degree of achievement of the objective. The criteria in general are in conflict, for instance a VoD with a higher quality is without a doubt more expensive than another VoD service with a normal or bad QoS. The Visual PROMETHEE tool put in evidence conflict between different criteria. PROMETHEE (Preference Ranking Organization METHod for the Enrichment of Evaluations) and Graphical Analysis for Interactive Aid (GAIA) techniques (Brans and Mareschal, 1994) (Mareschal et al., 1984) (Brans and Vincke, 1985), these methods are among the most widely used multicriteria decision aid methods, the software assist stakeholders in the evaluation of QoS evaluation, procedures, products, and so on, it helps to generate global quality scores by defining multiple evaluation criteria. A full quality diagnostic can be done through the GAIA analysis.

Visual PROMETHEE software is the solution that responds the best to our conditions. Besides evaluating numerous decisions related to different criteria in conflict, the software recognizes the most adequate decision and rank decisions from the best to the worst.

\subsection{Service Broker Architecture}

Figure 2 depicts our proposed architecture which is composed of IMS entities, service provider, modules to manage the SLA contract, ontology module containing SLA contract ontology component, service selection component in which the MCDM method is integrated. The architecture also includes databases with different information like consumer prole and his preferences. Based on user performances and old SLAs, Service Broker can identify the best service.

Service Broker has an important role which is to interface between service providers and consumers. First of all, it retrieves the requests, looks for the best offer available; negotiate the SLA contract content to at the end deliver the service to the consumer. Like we mentioned before, Service Broker proceeds to a negotiation phase after choosing the right service, proposals and alternative proposals are sent between the parties involved in the operation. The proposed decision architecture allows the customers to specify the weight of each negotiated quality attribute.

Our approach differs from other approaches that carry out SLA negotiation with several service providers, and then select the one with the best offer. It first selects the best suitable service provider based on the knowledge of the service broker on the providers' propositions. Then, it negotiates with the selected service provider the SLA terms in several rounds until an agreement reached or not.

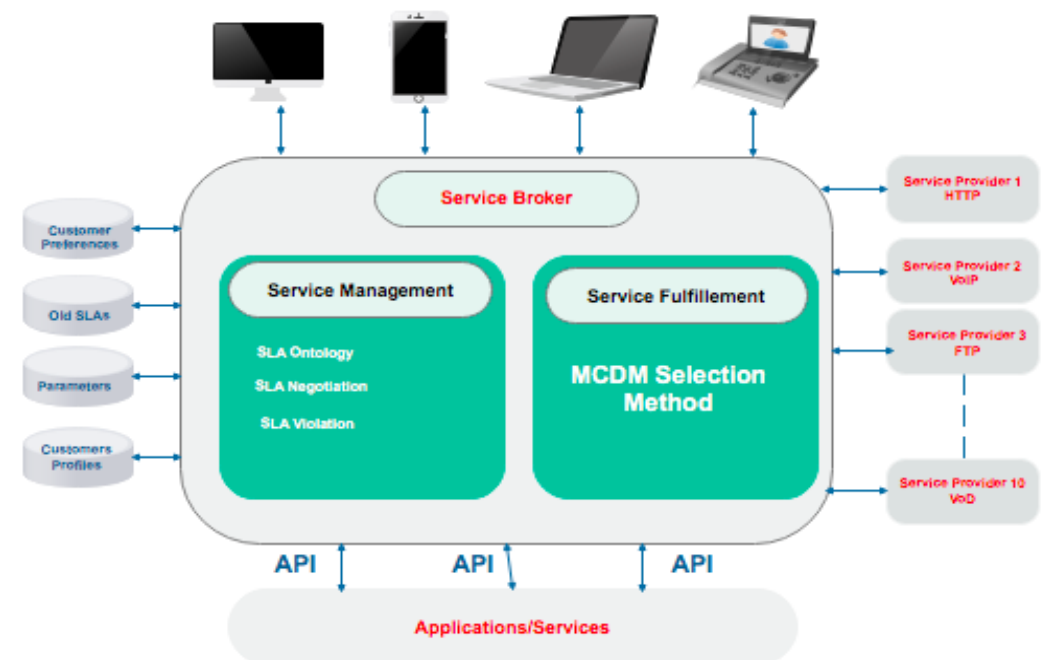

Figure 2. Service Broker Architecture 
Service Broker starts with verifying the consumer prole which can be platinum, gold, silver or bronze. He checks after that the type of the service wanted by the consumer, whether it is an IPTV, VoD (Video on Demand), VoIP (Voice over IP), etc. Right after that, Service Broker looks for the service provider that responds to the consumer QoS requirements, he executes the MCDM module to rank services and select the right one. After that, he goes on a negotiation process, creates the SLA contract and sends the required service to the consumer. All agreement and different rules are predefined in line with the ontology module.

To achieve an agreement about security, price, and other features, a module responsible of SLA management executes several tasks to do so. However, SLA violation module imposes penalties to service providers that does not respect the clauses mentioned in the SLA agreement contract.

\subsection{SLA Based Ontology}

We already mentioned that consumers face problems when it comes to express their needs. The heterogeneity appears in the definition of the same concept or requirement by different languages and terminologies. The use of ontology proved that it improves the identification and the detection of suitable services. The figure below depicts our SLA contract using protégé tool for ontology. The hierarchy begins from SLA Contract class that includes subclasses which are, Customer, Service provider and Contract term, and description of each subclass in included.

In a generic ontology describing SLA, consumer specifies his needs in terms of QoS criteria. However, service provider species the price of the required service and the QoS values that is committed to provide. The terms of the contract are identified by the Contract term attribute. This solution hides the heterogeneity between involved parties, it is a semantic proposition that share one vocabulary defining the needs and the offers, it also determines penalties on providers in case of violation detection of the SLA contract.

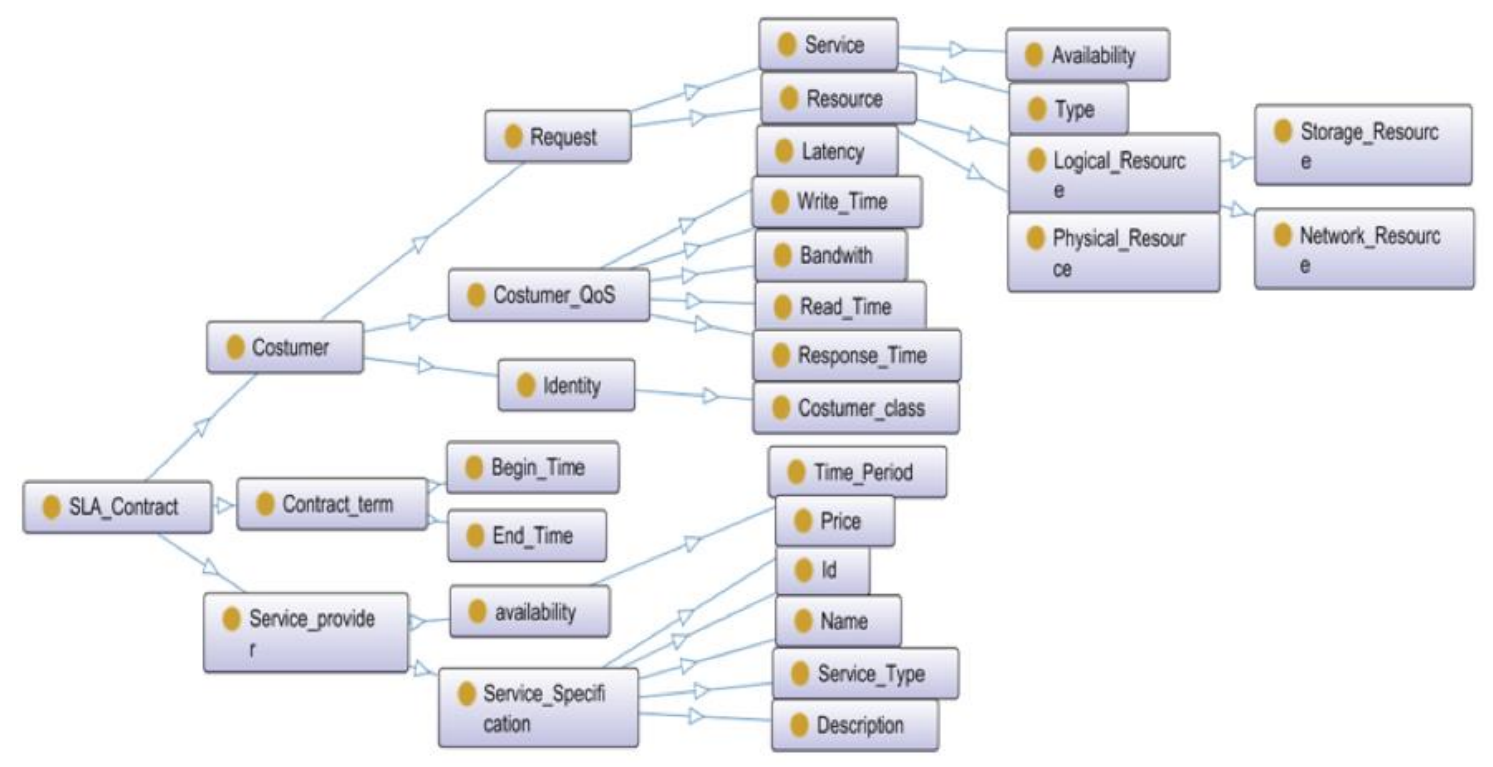

Figure 3. Architecture Ontology hierarchy of SLA Contract in Protégé

Most of users of IMS services are not familiar with IMS network; they may not be able to express consistent requests. We intended to verify the semantic consistency of the request before starting the selection operation that respond to the consumer needs. At this stage, Service Broker studies the request, analyses if it respects the ontology form, if the request is well written, then the Service Broker continues his process, however if the request is written in a bad way, Service Broker then alerts and asks the consumer to reword the request.

The customer's requirements include the request from the customer where the customer must specify its resource requirements, whether it is network resources or storage resources. In order to create entities such as virtual data centers, we need to define links between different resources (Figure 4). 


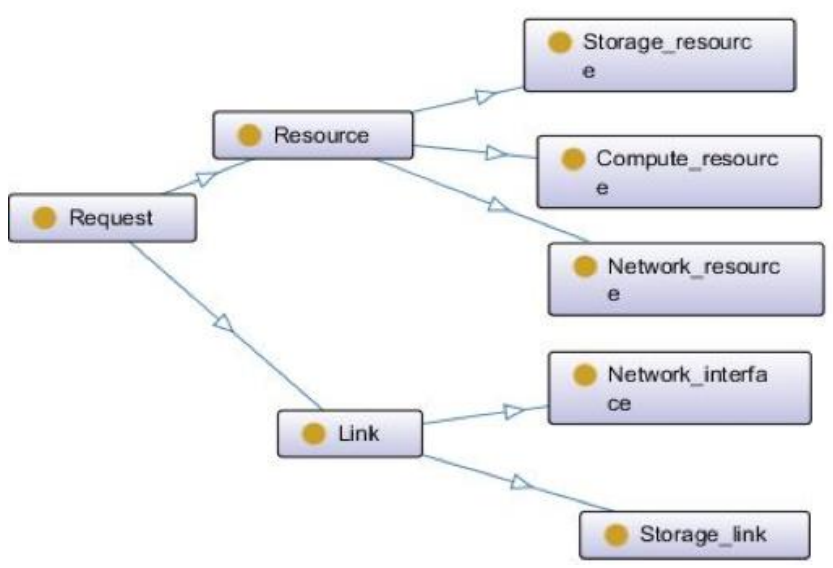

Figure 4. Service request

There are two types of links: the storage link connecting a compute resource to a storage resource, and the network interfaces linking the compute resources to the network resources. The customer requirements also contain some constraints that have a significant impact on the choice of the supplier, so the QoS required by the customer is an important criterion in the SLA specifications. SLA criteria can be classified into functional properties that define properties that directly influence quality of service such as cost, and non-functional properties that are rarely explicitly described in a customer request, but can be used as quality indicators such as reliability and trust.

\subsection{Service Provisioning and Negotiation Process}

Supplying a service goes through different steps, starting with the expressing the consumer requirements and expectations, SLA ontology contract is created based on a generic model already defined in the ontology module to allow to consumers to express their requests in a comprehensible way. The selection of o potential service provider is the next step; a negotiation process is required if it is needed to finalize the agreement and provide the service. Different forms can be taken in a negotiation process between stakeholders.

The negotiation process starts with the service broker after he receives the request from the customer to find an appropriate service provider that respond to the customer requirements and needs. After verifying the authentication, the service broker requests the customer prole to select the suitable service provider. The broker manager determines if the service provider founded can meet or not the customer needs.

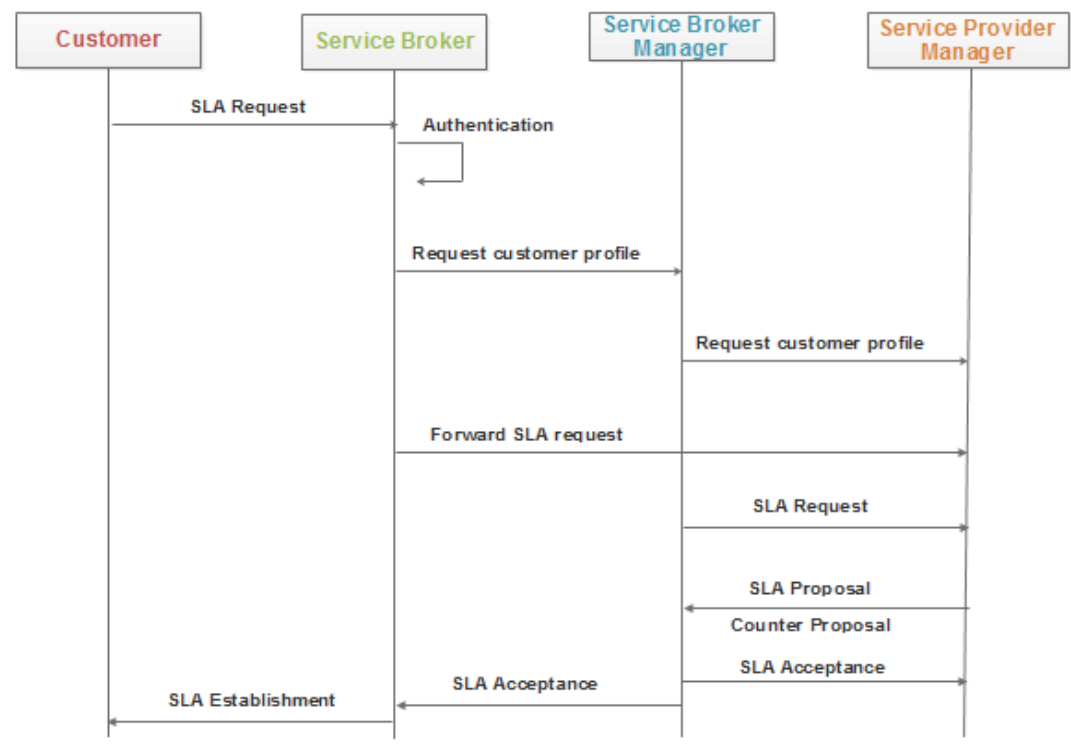

Figure 5. SLA Negotiation Process 
The decision is made based on the customer prole. The broker manager forwards the SLA request to the provider manager where proposal and counter proposal are made. The service broker manager analyses the proposal and if he accepts the service provider offer, an SLA confirmation is sent to the service broker who sent it to the customer. Otherwise, it rejects the offer and waits the customer proposal with other terms. If the involved parties approve on the different conditions and terms, the customer can enjoy his service respecting the terms of the QoS.

\section{SLA Management and Monitoring Architecture}

To ensure consumer loyalty, service providers are forced to diversify their services and offer a good QoS especially when it comes to multimedia services and real time applications. Several tools exist and different solutions were proposed to help to alleviate most congestion problems. However, many times there is just too much traffic for the bandwidth supplied. Managing the QoS mechanism with SLA negotiation process in network context to provide a determined QoS to services on demand and real times service proves complicated. To overcome this problem, we have already proposed the integration of New Generation Operations Systems and Software (NGOSS) framework to analyze the network and service activities in IMS network architecture on which we will test our solution. Our proposition aims to manage IMS services with QoS correction in case of degradation.

3GPP specifications provide a basic architecture for IMS network with a mechanism for QoS management, which ensures an adequate level of service compared to service supply in traditional Internet. However, the IMS services need to be monitored and managed by a set of mechanisms and methods of high level and that take into considerations the constraints of the business enterprise.

The NGOSS which is a set of operations to help service providers manage their business contains different frameworks that we used to manage our network. Among them we quote enhanced Telecom Operations Map (eTOM), which is a business process framework for telecom service providers in the telecommunications industry. The technical content of eTOM is more mature lately, with an increasing emphasis on guidelines for its application and usage. It is characterized by a hierarchy of process definitions and a common language for business processes. eTOM processes manage the most important tasks of an operator, however, to our knowledge, there is no real implementation for IMS network using eTOM specifications with Service Broker task, these still standard for all type of networks. The model describes the required business processes of service providers, and denes key elements and how they should interact.

eTOM contains 3 basic operations: fulfillment, assurance and billing. The fulfillment is done by the operator. However, the assurance process is used to implement proactive and reactive maintenance activities to ensure that services provided to consumers are readily available and they are satisfying, it also monitors the status of resources and performance to detect problems, the assurance processes manage also SLA contracts and service performance and send the report to the consumer.

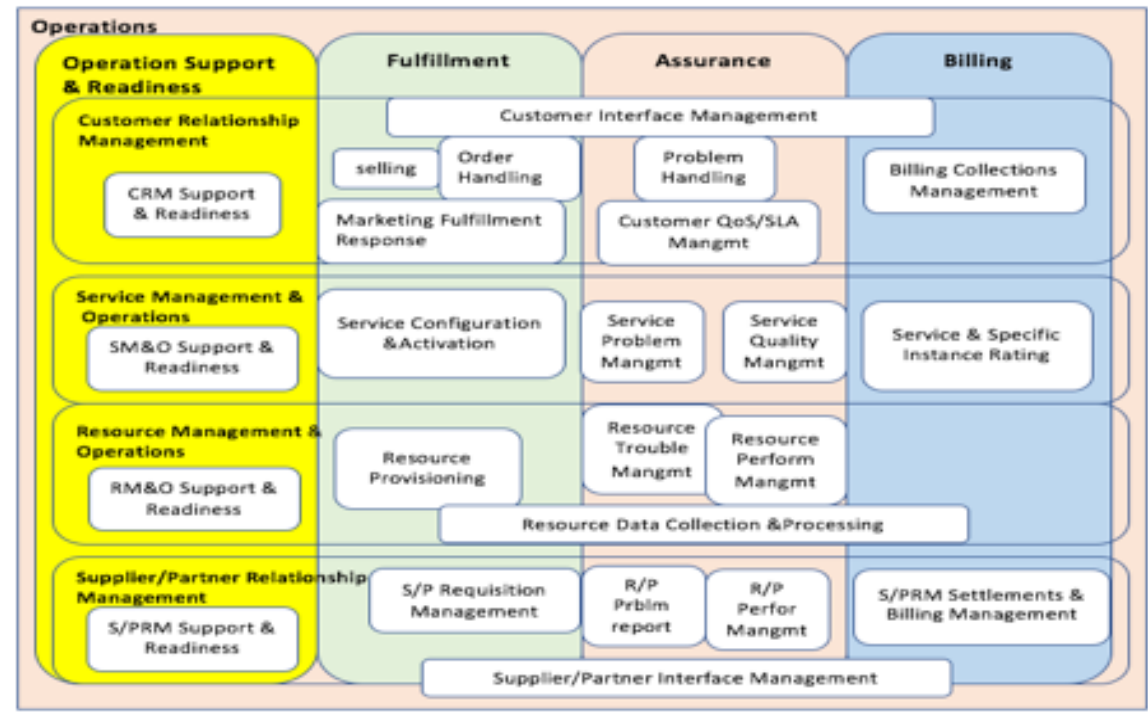

Figure 6. eTOM Business Process Framework Level 1 
The execution workflow of the contract describes scenarios and a lot of interactions between business processes and different information to help managing the execution and violation detection. As we already mentioned, any transaction between consumers and providers is in accordance with the SLA which is done in eTOM context through some steps to manage the network in a better way which are:

- Ordering: The consumer request goes through steps by selecting specified processes which retrieve information messages about the status of the request.

- Normal Execution of SLA: is a normal state of service delivery without violation occurrence. SLA verification requires a mapping between Key Performance Indicators (KPIs) and Key Quality Indicators (KQIs). KPI which contains only measurement of one metric, this metric is a calculated and aggregated measure of activities of SLA process, and KQI measures quality which is a degree of excellence; it gives a measurement of a particular aspect of the performance of the service. Like mentioned in the TMForum by Kelly (2003), KQI is supported by KPI, and KPI is supported by network performance data from network elements.

- Normal Execution of SLA with violation: The execution follow the same pathway of SLA verification until detection thresholds are exceeded for a specified period or failure of a supply component and thus the correction mechanisms will be launched to correct QoS degradation.

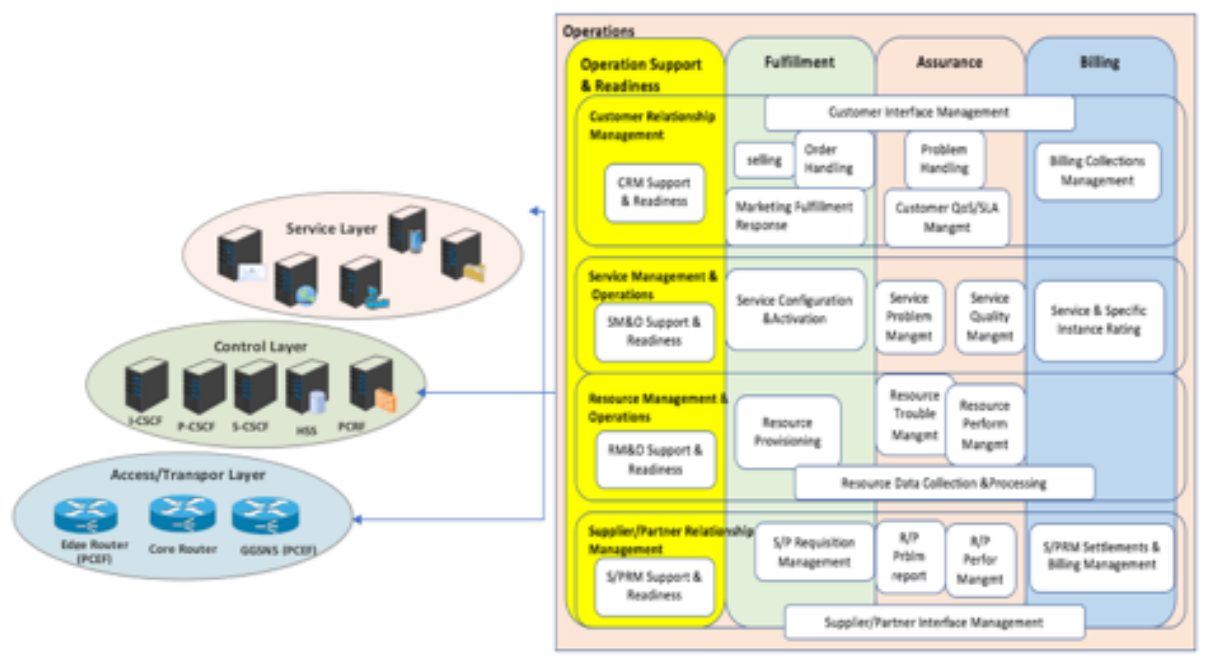

Figure 7. Processes operating in the SLA verification

Like we have already precise in a previous work (Raouyane et al., 2017), communications between IMS network and eTOM framework is done via TCP/IP, and XML. The integration of eTOM framework brings insights to provisioning and negotiation services in NGN networks. The management system of SLA improves the monitoring and specifies service performance measurements for efficient contract and to better manage the network. The interaction is a link between business processes and network entities.

\section{Results}

In order to verify and show some results, we have conducted a representative simulation used by the outranking method of visual PROMETHEE which evaluates the features. A pairwise comparison of the different action is the principle of the software.

PROMETHEE starts with comparing actions with each other's. It is done by computing a Multi-Criteria preference index in the following way (Mareschal, 2013).

$$
\prod(a, b)=\sum_{j=1}^{K} w_{j} * P_{j}(a, b)
$$

- $W_{j}>0 \quad$ is the normalized weight allocated to criterion $\mathrm{f}_{\mathrm{j}}$ (the more important $\mathrm{f}_{\mathrm{j}}$ the larger $\mathrm{w}_{\mathrm{j}}$ ).

- $\quad P_{j}(\mathrm{a}, \mathrm{b})$ is the value of preference function for criterion $\mathrm{f}_{\mathrm{j}}$ when action ' $\mathrm{a}$ ' is compared to action ' $\mathrm{b}$ '. 
- $\Pi(a, b)$ much ' $a$ ' is preferred to ' $b$ ' taking into account all the criteria and their weights.

With normalized weights, $\mathrm{P}(\mathrm{a}, \mathrm{b})$ is a number between 0 and 1 . It expresses how much ' $\mathrm{a}$ ' is preferred to ' $\mathrm{b}$ ' taking into account all the criteria and their weights.

As we can see from figure 8, phi+ which means the leaving flow and it is in comparison with phi- which is the entering flow. The best scores are upwards; the middle column matches the net flow (phi- scores).

To validate our testbed, and from the context of our work, we proposed four different IMS service providers, on the other hand, five criteria have been defined in order to choose and select the best service provider, the criteria are the price, Response Time, Read Performance, Latency, and Write Performance.

On the left side, we see the ranking of the actions according to Phi+, and on the right side we can see the ranking of the actions according to Phi- (Mareschal, 2013). For each action or criterion, a line is drawn from its Phi+ to its Phi- score. From the figure, when a line is totally on top of another one, it means that the corresponding action is better on the Phi+ and Phi- scores. For this reason, this action is preferred to other actions. In our case, Service Provider 2 is preferred to all the other service providers. In the middle of the ranking, Service provider 1 and Service provider 3 are very close to each other. However, Service Provider 4 is at the bottom of the raking, which means it is the worst provider to choose.

From this study, we can know the best provider to select which is provider 2, named in the Visual PROMETHEE Service P 2.

In the same concept, Figure 9 illustrates the Walking Weight, where we can manipulate the weights of any criterion and verify after that the impact on the analysis.

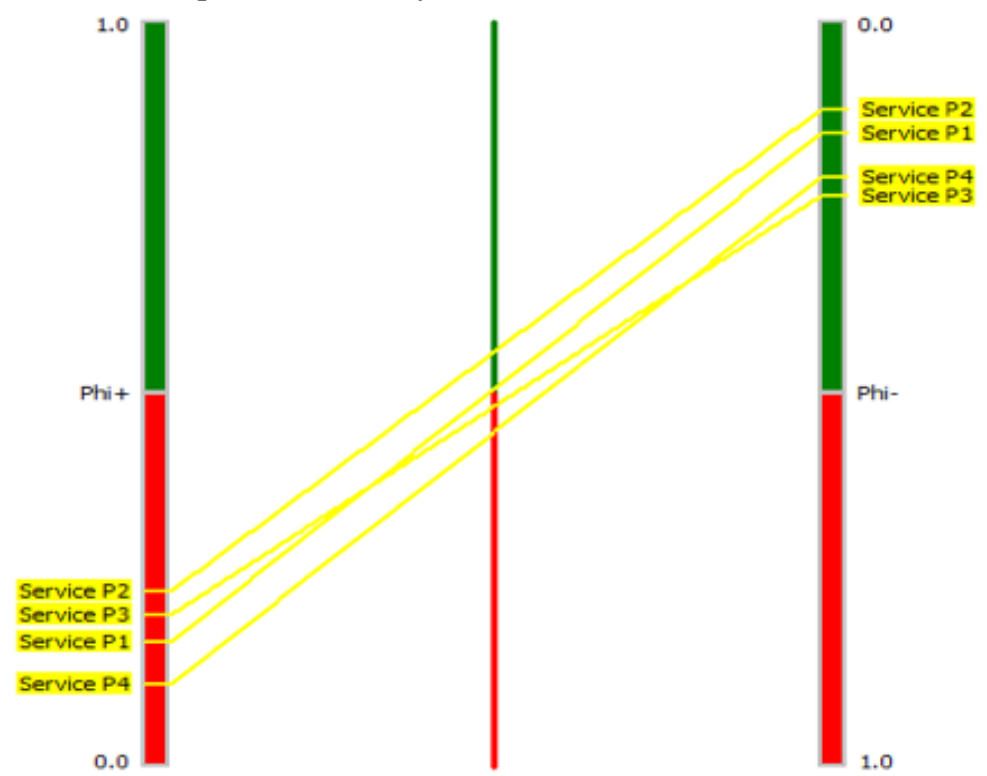

Figure 8. PROMETHEE Partial Ranking of our use case

Like we already mentioned, some criteria are to maximize, other are to minimize. From figure 9, we can see preference functions and weights have been associated to the criteria. In our case, all the weights have been set to equal values (which means $\mathrm{w}_{\mathrm{j}}=1$ where $\mathrm{j}=1,2 \ldots, 5$ ) without well-established priorities. 

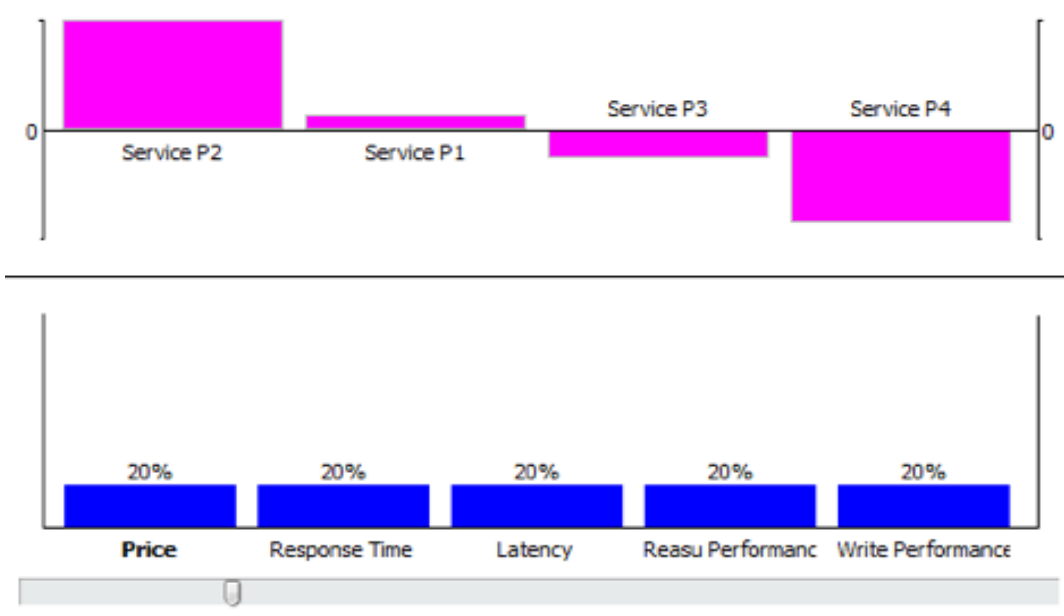

Figure 9. Walking weights of criteria

The rank of the actions in our test bed is from the worst to the best. The figure above includes two graphics; the upper one illustrates the Phi net flow scores for the actions in activity, in contrast to the lower one, which illustrates the relative weights of the criteria in percent unit.

However, Figure 10 illustrates the PROMETHEE network representation. Actions are represented by nodes and arrows are drawn to indicate preferences. For this reason, incomparability is easy to detect (Mareschal, 2013). The software uses an enhanced network representation; instead of drawing the nodes at arbitrary locations the relative positions of the actions in the software are used. The network representation, preferences are indicated by arrows; this makes it very straightforward to appreciate the proximity between actions and thus the degrees of incomparability in the partial ranking.

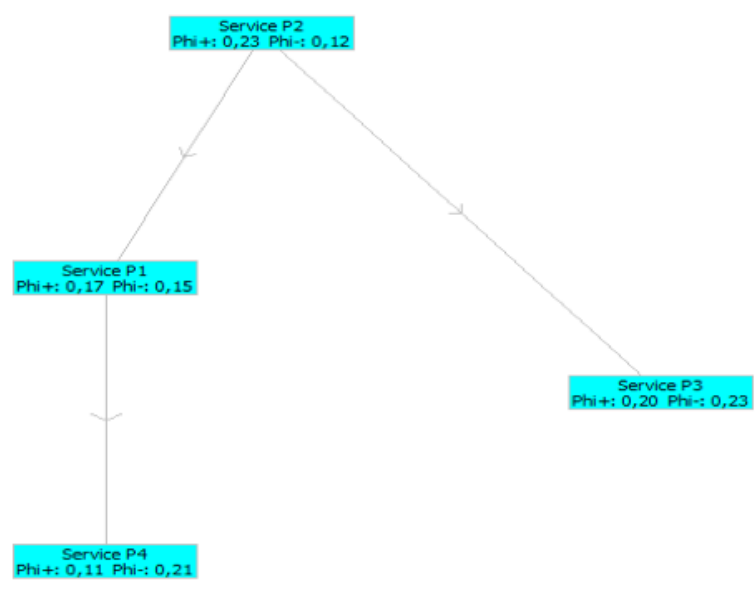

Figure 10. Network representation of actions

\section{Conclusions}

Given the expansion of the use of the Internet, multimedia services are widely adopted, each provider needs to be distinguished, they face challenges and concurrence to sell their services and satisfy consumers. In this work, we studied the ontology of the SLA to avoid the heterogeneity issue between different terminologies expressing the same requirement or request. The proposed architecture allows the negotiation of the SLA terms on behalf of consumers. Our presented architecture relies on Service Broker architecture which mediates between consumers and providers to select the appropriate service provider for the consumer. For this reason, we took a scenario with different actions and criteria using visual PROMETHEE software to demonstrate our approach. Also, a network management with NGOSS framework-based is proposed in this work in IMS environment. 


\section{References}

Andrieux, A., Czajkowski, K., Dan, A., Keahey, K., Ludwig, H., Nakata, P. J., .. Xu, M. (2007). Web services agreement specification (WS-Agreement). Open grid forum, 128(1), 216.

Baset, S. A. (2012). Cloud SLAs: present and future. ACM SIGOPS Operating Systems Review, 46(2), 57-66. https://doi.org/10.1145/2331576.2331586

Berbner, R., Spahn, M., Repp, N., Heckmann, O., \& Steinmetz, R. (2006). Heuristics for qos-aware web service composition. In 2006 IEEE International Conference on Web Services (ICWS'06) (pp. 72-82). https://doi.org/10.1109/ICWS.2006.69

Bermejo, J. (2007). A simplified guide to create an ontology. Madrid University, 1-12.

Bouyssou, D. (2009). Outranking methods. Encyclopedia of optimization, 2887-2893. https://doi.org/10.1007/978-0-387-74759-0_495

Brans, J. P., \& Mareschal, B. (1990). The PROMETHEE methods for MCDM; the PROMCALC, GAIA and BANKADVISER software. In Readings in multiple criteria decision aid (pp. 216-252). Springer, Berlin, Heidelberg. https://doi.org/10.1007/978-3-642-75935-2_10

Brans, J. P., \& Mareschal, B. (1994). The PROMCALC \& GAIA decision support system for multicriteria decision aid. Decision support systems, 12(4-5), 297-310. https://doi.org/10.1016/0167-9236(94)90048-5

Brans, J. P., \& Vincke, P. (1985). Note-A Preference Ranking Organisation Method: (The PROMETHEE Method for Multiple Criteria Decision-Making). Management science, 31(6), $647-656$. https://doi.org/10.1287/mnsc.31.6.647

Chen, Y. M., \& Huang, P. N. (2009). Agent-based bilateral multi-issue negotiation scheme for e-market transactions. Applied Soft Computing, 9(3), 1057-1067. https://doi.org/10.1016/j.asoc.2009.02.008

Chevaleyre, Y., Dunne, P. E., Endriss, U., Lang, J., Lemaītre, M., Padget, J., ... \& Sousa, P. (2005). Multiagent resource allocation. The Knowledge Engineering Review, 20(2), 143-149. https://doi.org/10.1017/S0269888905000470

Dan, A., Davis, D., Kearney, R., Keller, A., King, R., Kuebler, D., .. \& Youssef, A. (2004). Web services on demand: WSLA-driven automated management. IBM systems journal,43(1), 136-158. https://doi.org/10.1147/sj.431.0136

Di Modica, G., Tomarchio, O., \& Vita, L. (2009). Dynamic SLAs management in service oriented environments. Journal of Systems and Software, 82(5), 759-771. https://doi.org/10.1016/j.jss.2008.11.010

Dyer, J. S. (2005). MAUT-multiattribute utility theory. In Multiple criteria decision analysis: state of the art surveys (pp. 265-292). Springer, New York, NY. https://doi.org/10.1007/0-387-23081-5_7

Fakhfakh, I. (2015). Semantic based cloud broker architecture optimizing users satisfaction (Doctoral dissertation, Evry, Institut national des télécommunications).

Fishburn, P. C. (1967). Methods of estimating additive utilities. Management science, 13(7), $435-453$. https://doi.org/10.1287/mnsc.13.7.435

Garg, S. K., Versteeg, S., \& Buyya, R. (2011, December). Smicloud: A framework for comparing and ranking cloud services. In 2011 Fourth IEEE International Conference on Utility and Cloud Computing (pp. 210-218). https://doi.org/10.1109/UCC.2011.36

Haddar, I., Raouyane, B., \& Bellafkih, M. (2017, July). Generating a service broker framework for service selection and SLA-based provisioning within network environments. In 2017 Ninth International Conference on Ubiquitous and Future Networks (ICUFN) (pp. 630-365). https://doi.org/10.1109/ICUFN.2017.7993868

Hasselmeyer, P., Qu, C., Schubert, L., Koller, B., \& Wieder, P. (2006). Towards autonomous brokered SLA negotiation. Exploiting the Knowledge Economy-Issues, Applications, Case Studies, 3.

Ishizaka, A., \& Nemery, P. (2013). Multi-Criteria Decision Analysis: Methods and Software. https://doi.org/10.1002/9781118644898

Keeney, R. L., \& Raiffa, H. (1993). Decisions with multiple objectives: preferences and value trade-offs. Cambridge university press. https://doi.org/10.1017/CBO9781139174084

Keller, A., \& Ludwig, H. (2003). The WSLA framework: Specifying and monitoring service level agreements for 
web services. Journal of Network and Systems Management, 11(1), 57-81. https://doi.org/10.1023/A:1022445108617

Kelly, M. B. (2003). Report: The telemanagement forum's enhanced telecom operations map (etom). Journal of Network and Systems Management, 11(1), 109. https://doi.org/10.1023/A:1022449209526

Lai, G., Li, C., \& Sycara, K. (2006). Efficient multi-attribute negotiation with incomplete information. Group Decision and Negotiation, 15(5), 511-528. https://doi.org/10.1007/s10726-006-9041-y

Lamanna, D. D., Skene, J., \& Emmerich, W. (2003, January). Slang: A language for defining service level agreements. In NINTH IEEE WORKSHOP ON FUTURE TRENDS OF DISTRIBUTED COMPUTING SYSTEMS, PROCEEDINGS (pp. 100-106).

Lee, C. C., \& Ferguson, M. J. (2010). To reveal or not to reveal? Strategic disclosure of private information in negotiation. European Journal of Operational Research, 207(1), 380-390. https://doi.org/10.1016/j.ejor.2010.04.013

Ma, Y. B., Jang, S. H., \& Lee, J. S. (2011, April). Ontology-based resource management for cloud computing. In Asian Conference on Intelligent Information and Database Systems (pp. 343-352). Springer, Berlin, Heidelberg. https://doi.org/10.1007/978-3-642-20042-7_35

Mareschal, B. (2013). Visual PROMETHEE 1.4 manual. Visual PROMETHEE, 1.

Mareschal, B., Brans, J. P., \& Vincke, P. (1984). PROMETHEE: A new family of outranking methods in multicriteria analysis (No. 2013/9305). ULB--Universite Libre de Bruxelles.

Medjahed, B., \& Atif, Y. (2007). Context-based matching for web service composition. Distributed and Parallel Databases, 21(1), 5-37. https://doi.org/10.1007/s10619-006-7003-7

Menascé, D. A., Casalicchio, E., \& Dubey, V. (2010). On optimal service selection in service oriented architectures. Performance Evaluation, 67(8), 659-675. https://doi.org/10.1016/j.peva.2009.07.001

Mendiratta, V. B., \& Pant, H. (2007, December). Reliability of IMS architecture. In 2007 Australasian Telecommunication Networks and Applications Conference (pp. 1-6). IEEE. https://doi.org/10.1109/ATNAC.2007.4665302

Navarro, M., Donoso, Y., \& Rodriguez, V. (2010, June). An IMS architecture with QoS parameters for flexible convergent services. In The IEEE symposium on Computers and Communications (pp. 640-645). IEEE. https://doi.org/10.1109/ISCC.2010.5546770

O'sullivan, J., Edmond, D., \& Ter Hofstede, A. (2002). What's in a Service?. Distributed and Parallel Databases, 12(2-3), 117-133. https://doi.org/10.1023/A:1016547000822

Parra-Hernandez, R., \& Dimopoulos, N. J. (2005). A new heuristic for solving the multichoice multidimensional knapsack problem. IEEE Transactions on Systems, Man, and Cybernetics-Part A: Systems and Humans, 35(5), 708-717. https://doi.org/10.1109/TSMCA.2005.851140

Raouyane, B., Khairi, S., Haddar, I., \& Bellafkih, M. (2017, May). NGN Management with NGOSS Framework-Based IMS Use Case. In International Symposium on Ubiquitous Networking (pp. 166-178). Springer, Cham. https://doi.org/10.1007/978-3-319-68179-5_15

Roy, B. (1990). The outranking approach and the foundations of ELECTRE methods. In Readings in multiple criteria decision aid (pp. 155-183). Springer, Berlin, Heidelberg. https://doi.org/10.1007/978-3-642-75935-2_8

Silaghi, G. C., ŞErban, L. D., \& Litan, C. M. (2012). A time-constrained SLA negotiation strategy in competitive computational grids. Future Generation Computer Systems, 28(8), 1303-1315. https://doi.org/10.1016/j.future.2011.11.002

Sulaiman, M. S., Nordin, S., \& Jamil, N. (2017). An object properties filter for multi-modality ontology semantic image retrieval. Journal of Information and Communication Technologuy, 16(1), 1-19.

Tarkoma, S., Prehofer, C., Zhdanova, A. V., Moessner, K., \& Kovacs, E. (2007). SPICE: Evolving IMS to next generation service platforms. In 2007 International Symposium on Applications and the Internet Workshops (pp. 6-6). https://doi.org/10.1109/SAINT-W.2007.99

ur Rehman, Z., Hussain, O. K., Parvin, S., \& Hussain, F. K. (2012). A framework for user feedback based cloud service monitoring. In 2012 Sixth International Conference on Complex, Intelligent, and Software Intensive Systems (pp. 257-262). https://doi.org/10.1109/CISIS.2012.157 
Wang, S., Zheng, Z., Sun, Q., Zou, H., \& Yang, F. (2011). Reliable web service selection via QoS uncertainty computing. International Journal of Web and Grid Services, 7(4), 410-426. https://doi.org/10.1504/IJWGS.2011.044696

Wu, L., Garg, S. K., Buyya, R., Chen, C., \& Versteeg, S. (2013). Automated SLA negotiation framework for cloud computing. In 2013 13th IEEE/ACM International Symposium on Cluster, Cloud, and Grid Computing (pp. 235-244).

\section{Copyrights}

Copyright for this article is retained by the author(s), with first publication rights granted to the journal.

This is an open-access article distributed under the terms and conditions of the Creative Commons Attribution license (http://creativecommons.org/licenses/by/4.0/). 